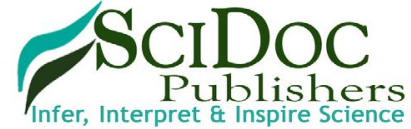

International Journal of Natural Disasters \& Health Security (IJNHS)

ISSN 2572-7540

\title{
Emergency Preparedness for Communicable Diseases in Public Hospitals in the Kumasi Metropolis of the Republic of Ghana
}

Research Article

Gborgblorvor $\mathrm{D}^{1}$, Opare-Addo $\mathrm{MN}^{1}$, Edzeamey $\mathrm{FJ}^{2 *}$, Wiredu $\mathrm{OK}^{2}$, Sampong BB ${ }^{2}$, Asamoah GD ${ }^{3}$, Ako AK

${ }^{1}$ Department of Health Policy, Management and Economics, School of Public Health, Kwame Nkrumah University of Science and Technology, Ghana.

${ }^{2}$ Laboratory Department, Bekwai Municipal Hospital, Ghana.

${ }^{3}$ Laboratory Department, Akomaa Memorial SDA Hospital, Bekwai-Kortwia, Ghana.

${ }^{4}$ Department of Biomedical Sciences, School of Allied Health Sciences, University of Cape Coast, Ghana.

\section{Abstract}

Background: Emergencies are unpredictable hence when they occur, they lead to immediate demand on public health resources. The capability of affected institutions and countries to handle such situations can be compromised, exposing the deficiencies at the various levels of their emergency preparedness.

Objective: This study therefore sought to investigate the emergency preparedness and how communicable diseases are managed in public hospitals in the Kumasi Metropolis.

Materials and Methods: This was a cross-sectional descriptive study performed among health care workers in five public hospitals in the Kumasi Metropolis using stratified random sampling technique. A total of 450 health workers from the five hospitals were recruited for the study. However, 360 of them participated in the study. A self-administered structured questionnaire was administered which reflected the objectives of the study. A quantitative method was employed to show how the variables were related.

Results: The study revealed that the facilities have emergency preparedness plans and teams for handling disease outbreaks and also, there were treatment protocols present on the walls in the facilities. $85 \%$ of communicable diseases diagnosed were isolated and treated at the facility, $12 \%$ was referred and $3 \%$ of the cases were treated and sent home. $87 \%$ of respondents indicated that there were no separate and specific structures for admitting and treating communicable diseases

Conclusion: Public hospitals are well prepared for any disease outbreak in the Kumasi Metropolis.

Keywords: Emergency Preparedness; Management; Communicable Diseases; Public Hospitals.

Abbreviations: SARS: Severe Acute Respiratory Syndrome; CHRPE: Committee on Human Research, Publication and Ethics; OPD: Outpatient Department; MET: Medical Emergency Team; RRT: Rapid Response Team.

\section{Introduction}

Emergencies are unpredictable hence when they occur, they lead to immediate demand on public health resources. The capability of affected institutions and countries to handle such situations can be compromised, exposing the deficiencies at the various levels of their emergency preparedness [1]. The main goal of every health facility is to prevent or minimize the loss of lives during such occurrences [2]. This can be done by health facilities formulating a comprehensive emergency plan in accordance with their manpower and infrastructural resources that will meet the demand of such occurrences. Emergency management is the discipline of dealing with and avoiding both natural and man-made disasters [3]. In addition to avoiding disasters, emergency preparedness involves mitigation, preparedness, response and recovery in order to reduce the impact of the disaster. Emergency preparedness is done at all levels and should not be done haphazardly. Successful preparedness requires detailed planning and collaboration among all stakeholders. The potential for disasters to occur exists in all communities. In the light of aforementioned the health sector should always anticipate disaster and disease outbreaks and put in prevention and preparatory measures in place so as to reduce the

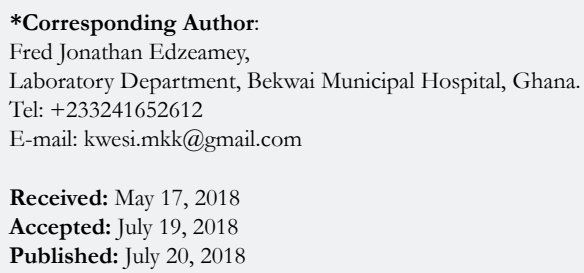

Copyright: Edzeamey $\mathbf{F J}^{\circ}$ 2018. This is an open-access article distributed under the terms of the Creative Commons Attribution License, which permits unrestricted use, distribution and reproduction in any medium, provided the original author and source are credited. 
impact of such occurrences. There are reports of disaster occurrences such as floods, drought, cyclones, earthquakes and infectious disease outbreak around the world, and that emergencies are totally unpreventable, but their impact can be minimized by effective planning.

Disease outbreaks can create a variety of hazards for health workers in the affected geographic area. Preparing before emergencies occurs plays an important role in ensuring that employers and workers have the necessary equipment, knowledge, skills and attitude to protect themselves and others. It is therefore very important every health facility has an emergency preparedness plan ready at all times.

New and emerging communicable diseases with the potential to cause severe epidemics or pandemics are increasingly prevalent, and therefore what previously passed for acceptable planning and diseases events are now re-evaluated [4]. These diseases endanger the lives of citizens and exacerbate social and political instability in affected countries and regions. Communicable diseases constitute a major public health problem all over the world, it has the potential to cause outbreaks and affect a large majority of the people $[5,6]$.

The spread of communicable diseases happens through airborne viruses or bacteria, also through contact with blood or other bodily fluid. It is spread through direct contact or close proximity as the causative bacteria or viruses are mostly airborne. They can be expelled from the nose and mouth of the infected person and inhaled by anyone in the vicinity of such diseases. The increase in the spread of infectious disease results from changes in human behaviour including lifestyle and land use patterns [7].

Globalization also increases the chances of an infectious disease in one country spreading rapidly to another country within a few days to weeks. This can be exemplified by Severe Acute Respiratory Syndrome (SARS) which within a matter of weeks spread in early 2003 from Guangdong province of China to rapidly infect individuals in some 37 countries around the world [8]. Infectious disease poses a rising global health threat and will complicate global security over the next 20 years [9].

Communicable diseases persist to be the major cause of illness and death worldwide but mortality and mobility rates for these diseases in developed countries decreased noticeably by end of the twentieth century. The decline in the industrialized countries can be associated to a lot of complex factors which include foreseeing and adequately preparing toward its occurrences, this includes better sanitation, availability of vaccines and antimicrobial drugs [10].

Richard Smith indicated that the development of an effective surveillance and response system probably is the most important way of reducing the impact of diseases outbreak [9]. Preparing before emergency occurs plays an important role in ensuring that employers and workers have the necessary equipment, knowledge, and skills and attitude to protect themselves and others [1].

It is necessary and important to detect disease outbreaks early in order to minimize the morbidity and mortality that are associated with it, and this can be done by timely implementation of disease prevention and control measures [11]. Nations, state, and local health departments are initiation disease surveillance systems with day by day analyses of hospital emergency department visits, ambulance dispatch calls etc., [11].

\section{Materials and Methods}

This was a cross-sectional descriptive study conducted among health care workers in five public hospitals in the Kumasi Metropolis using stratified random sampling technique. A total of 450 health workers from the five hospital (Kumasi South Hospital, Manhyia Government Hospital, Maternal \& Child Health Hospital, Suntreso Government Hospital and Tafo Government Hospital) were recruited for the study. However, 360 of them participated in the study. A self-administered structured questionnaire was administered which Comprised the demographic characteristics, communicable disease management, presence and placement of treatment protocols for communicable diseases, training and availability of respondents for management of communicable diseases, the department's staff trained for the management of communicable diseases, availability of separate and specific structures for admitting and treating communicable diseases and who respondents contact in communicable diseases crises. Whilst Medical Officers, Physician Assistants, Pharmacists and Pharmacy Technicians, all categories of Nurses, Biomedical and Laboratory Scientists, and Disease Control Officers were included in the study, health workers and management team members who decline to be included in the study, Clinical care staff that had worked for less than six months during the time of data collection at the facility, nonclinical care workers and staff who were on leave at the time of the study were excluded. The health workers were put into strata based on profession: Medical Officers were in one stratum, all categories of Nurses in other stratum, Physician Assistants/Medical Assistants in one stratum, Pharmacists and Pharmacy Technicians in one stratum, and Disease Control Officers formed one stratum, Biomedical and Laboratory Scientists also formed one stratum. A quantitative method was employed to show how the variables were related.

\section{Ethical Consideration}

Prior to the study, ethical approval was obtained from the Committee on Human Research, Publication and Ethics (CHRPE). A written approval was obtained from the management of the five hospitals and from the Kumasi Metropolitan Health Directorates before undertaking the study. Written informed consent for the study was obtained from respondents after the purpose of the study had been explained. Respondents were given at least 24 hours to decide to participate or opt out at any point in the study.

\section{Statistical Analysis}

Data was analysed using Epi Info software programme.

\section{Results}

The average participation of the five public health facilities recorded in this present study was 20\% with 360 respondents participating fully in the study. 


\section{Demographic characteristic of respondents}

$72(20 \%)$ of the respondents were from the Kumasi South Hospital, $65(18.1 \%)$ from the Manhyia Government Hospital, 75 (20.8\%) from the Maternal \& Child Health Hospital, 73 (20.3\%) from the Suntreso Government Hospital and 75 (20.8\%) of the respondents from the Tafo Government Hospital. It was revealed from this study that more than half the respondents $(55.8 \%)$ had worked in the health sector for less than 5 years and more than three fourth $(76 \%)$ of the respondents had worked at their various facilities for less than 5 years. Close to a quarter (23.1\%) had worked at their various facilities for between 5 and 15 years. Just about $1 \%$ had worked in the facilities for more than 15 years.

\section{Emergency preparedness for Communicable diseases}

The study revealed that there were emergency preparedness plan for communicable diseases in the public hospitals under consideration. $56 \%$ of the respondents from the five facilities indicated that the facilities had emergency preparedness plan specific for communicable diseases, $17 \%$ do not know of the presence of emergency preparedness plan specific for communicable diseases whilst $27 \%$ of the respondents said there are no emergency preparedness plan for communicable diseases. Also, $79 \%$ of the respondents were members of the emergency preparedness team of in their various facilities. The percentage of respondents in the category of professions in the emergency team for communicable diseases is illustrated in Figure 1 below.

From the study some members of the management were also members of the emergency team of the facilities, giving $79 \%$ of the respondents to be members of the emergency preparedness team. $(52 \%)$ indicated that management does not provide the needed support for the activities of the emergency preparedness team of the facilities.

However, 98\% of the respondents indicated that there were no emergency preparedness team specifically for communicable diseases in their various facilities.

\section{Management of communicable diseases}

It was also revealed in this study by respondent that most cases $(85 \%)$ of communicable diseases are isolated and treated at the facilities, $12 \%$ are however referred to the next level of care and $3 \%$ are treated and sent home. Majority of the respondents $(80 \%)$ affirmed that there were trained health workers for the management of communicable diseases in the hospitals under consideration whilst $20 \%$ stated otherwise. It was further established by this research that $89 \%$ of the staffs trained in the management of communicable diseases worked at the public health unit, with $9 \%$ working at the other units combined apart from 2\% who worked at the Out Patient Department (OPD). $87 \%$ of the respondents stated that there were no separate and specific rooms or structure for admitting and treating of communicable diseases in the facilities whilst $13 \%$ indicated that they have no idea about neither the presence nor existence of these rooms. Also, $65 \%$ of the respondents indicated that the staffs reported any communicable disease diagnosed in the facility to the Disease Control Officer, $25 \%$ said to the unit in-charge and $2 \%$ to the Medical Director of the facility.

It was also revealed that treatment protocols for the management of communicable disease cases were also available in the facilities and can be found on the walls of the facilities as indicated by $87 \%$ and $57 \%$ of the respondents respectively (Figure 2).

\section{Discussion}

From this study, some members of the management were also members of the emergency team of the facilities, giving $79 \%$ of the respondents to be members of the emergency preparedness team. One would then expect maximum support of management to the work of the emergency preparedness teams but that does not seem to be the case as more than half of the respondents $(52 \%)$ indicated that management does not support the activities of the emergency preparedness team of the facilities. Even though there were emergency teams in each facility, almost all the respondents $(98 \%)$ indicated that there was no emergency preparedness team specifically for communicable diseases in the facilities. Contrary to Jones et al., 2009's article that expects separate teams for the different emergencies that could happen in our health care facilities as their management would require a certain expertise. An example would be that Emergency Medical Team (MET) would require the expertise of an Internal Medicine Physician and a Rapid Response Team (RRT) for communicable diseases be led by a Public Health Physician with expertise in communicable diseases.

Figure 1. The percentage of respondents in the category of professions.

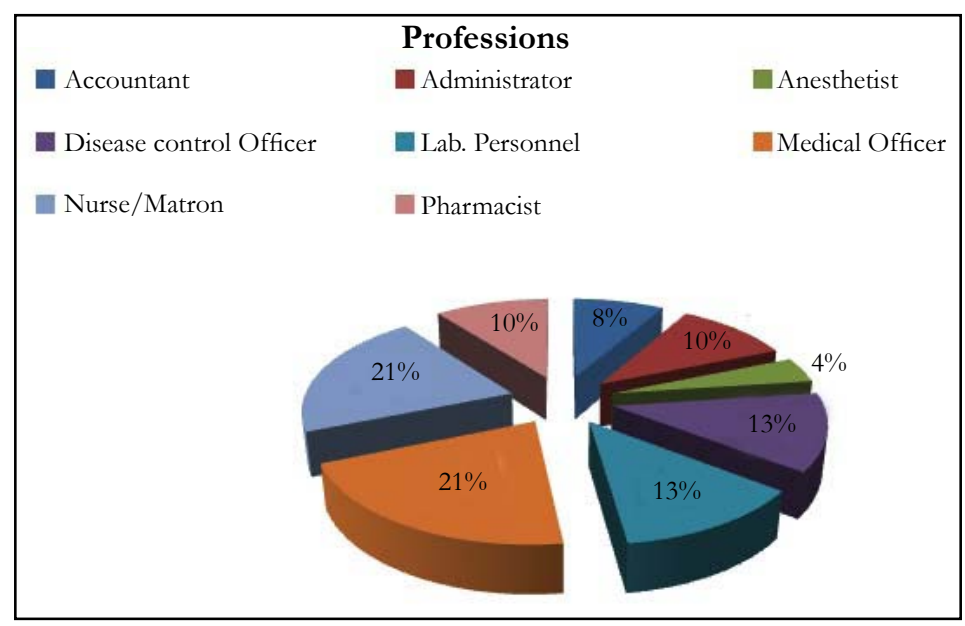


Figure 2. Presence and placement of treatment protocols for communicable disease.

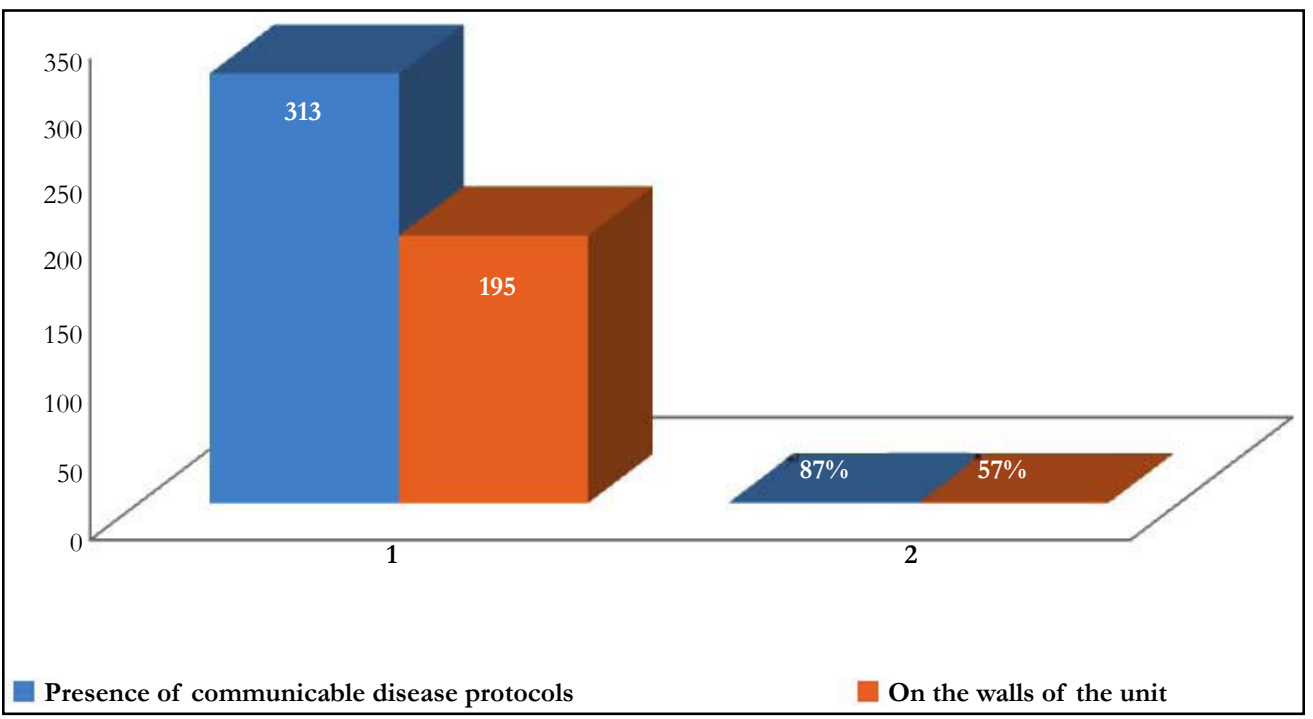

To determine the emergency preparedness level of health facilities in disaster management certain key areas needs to be put into consideration such as treatment guidelines, trained personnel plans, equipment and epidemiologic surveillance and these indicators seem to be in place in all the study facilities [1]. Staff had received training in the management of communicable diseases, there is treatment protocols readily available and could be found and assessable to all.

The study again recorded that $85 \%$ of the respondents indicated communicable diseases are isolated and treated; this is in accordance with the Ghana Health service treatment protocols to isolate and treat communicable disease at the diagnosed facility to prevent further spread of the disease.Health workers in the facilities were trained on the management of communicable diseases $(80 \%)$, and treatment protocols were also available in the facilities $(87 \%)$, it is considered a good practice as emphasized the importance of public health workers been knowledgeable in infectious disease management [12].

More than half $(56 \%)$ of the respondents from all five hospitals stated that emergency preparedness plan for communicable diseases could be found in the facilities and this is in agreement with a research indicating that emergency preparedness plan was very important and needed to confidently manage any emergency situation that may arise [13].

In the management of disease outbreaks and disasters, knowledge and skills plays an integral role in management and preparing for emergency situation as indicated by [14]. which was been practice in the facilities the study was conducted as $80 \%$ of the respondents affirmed to health receiving training on the management of communicable diseases.

\section{Conclusion}

There were emergency preparedness teams in all five hospitals, but none of the hospitals had an emergency preparedness team specifically for communicable diseases and also, the support from management was not adequate, even though some members of the management team were also members of the emergency preparedness teams.

Also the current practices of communicable disease management in the facilities were good as communicable disease cases were isolated and treated, treatment protocols for the management of communicable diseases are readily available in all facilities under consideration. This indicates that these facilities are readily prepared should there be any disease outbreak in their vicinity. However, the absence of separate and specific rooms for the admission and treatment of communicable diseases could be seen as a strong impediment to the smooth and effective management of communicable diseases in the unlikely event when there is an outbreak.

\section{References}

[1]. Peña-Orellana M, Rivera-Gutiérrez R, Gónzalez-Sánchez JA, Padilla-Elías N, Centeno HM, Alonso-Serra H, et al. Assessing Healthcare Facilities Preparedness for Mass Fatalities Incident. Int J Clin Med. 2013 Dec 18;4(12):525.

[2]. Connolly MA, Gayer M, Ryan MJ, Salama P, Spiegel P, Heymann DL. Communicable diseases in complex emergencies: impact and challenges. Lancet. 2004 Nov 27-Dec 3;364(9449):1974-83. PubMed PMID: 15567014.

[3]. Baird ME. The "phases" of emergency management. Background paper. Prepared for the Intermodal Freight Transportation Institute (ITFI) University of Memphis. Nashville: Vanderbilt Center for Transportation Research (VECTOR). $2010 \mathrm{Jan}$

[4]. Carpenter C, Wyman O. The economic and social impact of emerging infectious disease: mitigation through detection, research, and responsell. Retrieved July. 2008;21:2011.

[5]. Noah D, Fidas G. National intelligence estimate: the global infectious disease threat and its implications for the United States. Environ Change Secur Proj Rep. 2000 Summer;(6):33-65. PubMed PMID: 12296202.

[6]. Fonkwo PN. Pricing infectious disease: The economic and health implications of infectious diseases. EMBO Rep. 2008 Jul;9 Suppl 1:S13-7. doi: 10.1038/embor.2008.110. PubMed PMID: 18578017.

[7]. Kimball AM. Risky trade: Infectious disease in the era of global trade. Routledge; 2016 Apr 8.

[8]. Wang MD, Jolly AM. Changing virulence of the SARS virus: the epidemiological evidence. Bull World Health Organ. 2004 Jul;82(7):547-8. PubMed PMID: 15500287.

[9]. Smith RD. Responding to global infectious disease outbreaks: lessons from SARS on the role of risk perception, communication and management. Soc Sci Med. 2006 Dec;63(12):3113-23. PubMed PMID: 16978751.

[10]. López-Cuadrado T, Llácer A, Palmera-Suárez R, Gómez-Barroso D, Savulescu C, González-Yuste P, et al. Trends in infectious disease mortality rates, Spain, 1980-2011. Emerg Infect Dis. 2014 May;20(5):782-9. doi: 10.3201/ 
eid2005.131528. PubMed PMID: 24750997.

[11]. Kulldorff M, Heffernan R, Hartman J, Assunçao R, Mostashari F. A spacetime permutation scan statistic for disease outbreak detection. PLoS Med. 2005 Mar;2(3):e59. PubMed PMID: 15719066.

[12]. Chandler T, Qureshi K, Gebbie KM, Morse SS. On Academics: Teaching Emergency Preparedness to Public Health Workers: Use of Blended Learning in Web-Based Training. Public Health Rep. 2008 Sep-Oct;123(5):676-80.
PubMed PMID: 18828425.

[13]. Smith E. Emergency health care workers' willingness to work during major emergencies and disasters. Aust J Emerg Manage. 2007 May;22(2):21.

[14]. Wisniewski R, Dennik-Champion G, Peltier JW. Emergency preparedness competencies: assessing nurses' educational needs. J Nurs Adm. 2004 Oct;34(10):475-80. PubMed PMID: 15577671. 